\title{
Review
}

\section{The Effect of Locally Delivered Statins on Treating Periodontal Intrabony Defects: A Systematic Review and Meta-Analysis}

\author{
Khaled Sinjab, * Nouf Zimmo, * Guo-Hao Lin, ${ }^{* \dagger}$ Ming-Pang Chung, * Lujain Shaikh, * \\ and Hom-Lay Wang*
}

Background: Use of statins on adult patients with chronic periodontitis shows a positive effect on their periodontal status. However, effect of locally delivered statins on periodontal treatment has not yet been systematically analyzed. Hence, the present systematic review and meta-analysis aims to evaluate efficacy of statins on treating localized periodontal intrabony defects (IBDs).

Methods: An electronic search of three databases (PubMed, Embase, and Cumulative Index to Nursing and Allied Health Literature) between January 1, 1965 and March 1, 2016, and a hand search of peerreviewed journals for relevant articles were performed. Controlled randomized clinical trials (RCTs) and prospective studies with data on comparison between adjunctive locally delivered statin use to mechanical scaling and root planing (SRP) and placebo in each group, with minimum 10 participants and followup period of at least 6 months, were included.

Results: Ten studies, eight RCTs and two prospective studies, were included. Each study included 15 to 105 patients between 25 and 55 years of age. Statistical results were recorded; weighted mean difference (WMD) and confidence interval (CI) were calculated; and meta-analyses were performed for defect fill, probing depth (PD) reduction, and clinical attachment level (CAL) gain in both statin and placebo/no treatment groups. Overall analysis of defect fill presented WMD of $1.37 \mathrm{~mm}(95 \% \mathrm{CI}=0.96$ to 1.77 ; $P<0.0001)$, PD reduction presented WMD of $1.76 \mathrm{~mm}(95 \% \mathrm{CI}=1.04$ to $2.47 ; P<0.0001)$, and $\mathrm{CAL}$ gain presented WMD of $1.58 \mathrm{~mm}(95 \% \mathrm{CI}=0.89$ to $2.28 ; P<0.0001)$. However, comparison presented considerable heterogeneity among studies.

Conclusions: This systematic review and meta-analysis find that adjunctive use of locally delivered statins to mechanical SRP is beneficial to increasing bone fill percentage. Improved inflammatory and bleeding control as well as PD reduction and CAL gain are possible advantages to using these drugs in treating patients with periodontal IBDs. J Periodontol 2017;88:357-367.

\section{KEY WORDS}

Alveolar bone loss; hydroxymethylglutaryl-CoA reductase inhibitors; meta-analysis as topic; periodontitis; review literature as topic.

\footnotetext{
* Department of Periodontics and Oral Medicine, University of Michigan School of Dentistry, Ann Arbor, MI.

$\dagger$ Department of Surgical Sciences, Marquette University School of Dentistry, Milwaukee, WI.
} 
$\mathrm{P}$ eriodontitis is a chronic inflammatory and infectious disease of tooth-supporting tissues leading to both attachment loss (AL) and bone loss (BL), ${ }^{1}$ which eventually could lead to formation of intrabony defects (IBDs). These inflammatory conditions are caused by a combination of microbial plaque and unbalanced host immune responses ${ }^{2}$ producing proinflammatory cytokines and proteolyticderivative enzymes that accelerate $\mathrm{AL}$ and BL. ${ }^{3}$

Use of controlled-release, locally delivered antimicrobial agents in adjunct to mechanical treatments, especially when placed subgingivally targeting specific microorganisms, offers favorable results in treatment of localized periodontally destructed areas. ${ }^{4}$ According to the American Academy of Periodontology, ${ }^{5}$ results achieved in systematic reviews ranged between 0.25 and $0.5 \mathrm{~mm}$. Antimicrobial agents such as chlorhexidine gluconate (CHX), minocycline (MIN), doxycycline (DOX), metronidazole (MET), and tetracycline (TCN) are selected because of their sustained release and significant probing depth (PD) reduction or CAL gain when used in adjunct to SRP. ${ }^{6}$

Statins were first introduced as cholesterol-lowering drugs by inhibiting 3-hydroxy-3-methylglutaryl coenzyme A reductase, ${ }^{7}$ interfering in the cholesterol synthesis process in the liver, which lowers cholesterol and decreases incidence of cardiovascular disease. Each year they are among the most prescribed and top-selling drugs in the United States, especially in middle-aged adults. ${ }^{8}$ Statins have been reported to also possess anti-inflammatory properties, ${ }^{9}$ antioxidative features, ${ }^{10}$ antibacterial activities, ${ }^{11}$ and pleiotropic features, such as inhibiting release of proinflammatory mediators and matrix metalloproteinases (MMPs). ${ }^{12}$ Due to these characteristics, statins have been used for treating patients with periodontitis, ${ }^{13}$ aiming to reduce lipopolysaccharide (LPS)-induced MMP-1, MMP-8, and MMP-9 expressions in monocytes ${ }^{14}$ as well as expression of MMP-9 in osteoblastic cells. ${ }^{15}$

Statins have been found to have other functions in the oral cavity, such as inhibiting interleukin (IL)- $6^{16}$ and LPS-induced expression of proinflammatory genes, ${ }^{17}$ intercellular adhesion molecule- $1,{ }^{18}$ and nitric oxide synthase. ${ }^{19}$ They are also known to aid in reduction of protein prenylation by inhibiting the mevalonate pathway upstream of bisphosphonates in osteoclasts, eventually affecting their normal function, ${ }^{20}$ and exhibiting positive effect on proliferation and osteoblastic differentiation of periodontal ligament cells as seen in an in vitro study. ${ }^{21}$ In addition, statins have been found to stimulate bone morphogenetic protein 2 expression, ${ }^{22}$ which results in stimulation of osteoblast proliferation and bone formation.

Various animal studies reported success in bone regeneration when statins were applied locally. 23-26
More recently, one systematic review ${ }^{27}$ concluded that use of statins on adult patients $\geq 30$ years of age with chronic periodontitis (CP) showed positive effect on their periodontal status, and that concomitant administration of either systemic or locally delivered statins in adjunct to non-surgical therapy may help improve periodontal health. Results of another systematic review ${ }^{28}$ also indicated beneficial effects of statins and their potential to improve therapeutic effect during PD treatments. However, a lack of metaanalysis of the effect of statins on periodontal treatment has been identified. Therefore, the aim of the current study is to evaluate efficacy of locally delivered statins on treating localized periodontal IBDs.

\section{MATERIALS AND METHODS}

\section{Focused Question}

How effective are locally delivered statins on treating periodontal defects in patients with $\mathrm{CP}$ ? This question considers: 1) population: individuals with $\mathrm{CP}$ and periodontal IBDs; 2) intervention: use of locally delivered statin drugs in adjunct to mechanical scaling and root planing (SRP); 3) comparison: statin group compared with placebo/no treatment group; and 4) outcomes: changes in defect depth, PD reduction, clinical attachment level (CAL) gain.

\section{Selection Criteria}

Inclusion criteria were: 1) publications of domestic and international peer-reviewed literature; 2) articles written in English; 3) human controlled randomized clinical trials (RCTs) and prospective studies; 4) $\geq 6$-month follow-up; 5) $\geq 10$ patients participating in the study; and 6) articles reporting data on changes of defect depth, PD reduction, and/or CAL gain. Exclusion criteria were: 1) animal studies; 2) no definition of inclusion and/or exclusion criteria reported; 3) no specific information of drug administration; 4) insufficient information on adjunctive therapy during treatment period; and 5) retrospective cross-sectional studies, case series, or case reports.

\section{Search Strategy}

A literature search was performed at the University of Michigan School of Dentistry, Ann Arbor, Michigan, through hand search of peer-reviewed journals for relevant articles and electronic search of three different databases (PubMed, Embase, and Cumulative Index to Nursing and Allied Health Literature [CINAHL]) between January 1, 1965 and March 1, 2016, using the following terms for each database.

PubMed. ("Hydroxymethylglutaryl CoA Reductase Inhibitors"[mh] OR "reductase inhibitor"[tiab] OR "reductase inhibitors"[tiab] OR statin[tiab] OR statins[tiab] OR atorvastatin[tiab] OR fluvastatin[tiab] OR rosuvastatin[tiab]) AND ("periodontal diseases"[mh] OR periodontal[tiab] OR "bone regeneration" [mh] OR "peri-odontology"[tiab] OR mandible[tiab] OR 


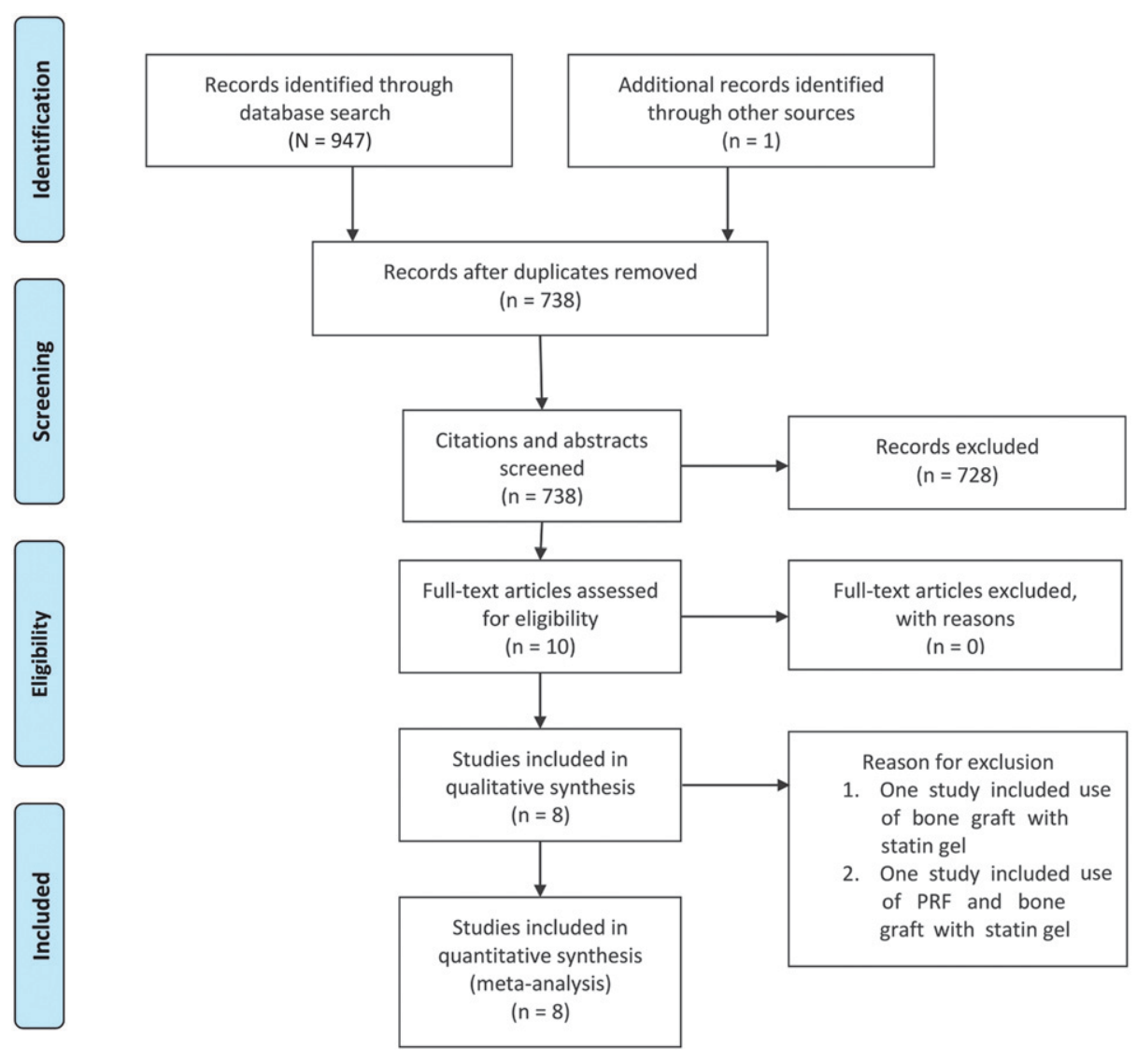

Figure 1 .

Flow diagram of literature search and screening process.

OR AB intrabony OR TI "intrabony" OR AB infrabony OR TI alveolar OR AB alveolar).

Two reviewers (NZ and MPC) independently performed the article search and selection process. They first examined the titles and abstracts of the articles and selected papers for full-text screening. Any disagreements between reviewers were resolved through discussion. The $\kappa$ value was introduced to examine interexaminer agreement.

\section{Data Extraction}

Articles fulfilling all inclusion criteria were selected for data extraction (Fig. 1).

\section{Risk of Bias (Quality) Assessment}

Criteria used to assess quality of the selected RCTs were modified from the RCT checklist of the Cochrane Center ${ }^{29}$ and the Consolidated Standards of Reporting Trials statement, ${ }^{30}$ which provided guidelines for the following parameters: 1) sequence generation; 2) allocation concealment method; 3) masking of examiner; 4) addressing in-

peridontitis[tiab] OR "tooth extraction"[tiab] OR furcation [tiab] OR intrabony[tiab] OR "intra-bony"[tiab] OR infrabony[tiab] OR "infra-bony"[tiab] OR alveolar[tiab]).

Embase. "hydroxymethylglutaryl coenzyme a reductase inhibitor"/exp OR "reductase inhibitor":ab, ti OR "reductase inhibitors":ab,ti OR statin *:ab,ti OR atorvastatin:ab,ti OR fluvastatin:ab,ti OR rosuvastatin:ab,ti AND ("periodontal disease"/exp OR periodont*:ab,ti OR "bone regeneration"/exp OR "peri odontology":ab,ti OR mandible*:ab,ti OR "tooth extraction":ab,ti OR "tooth extractions":ab,ti OR furcation*:ab,ti OR intrabony:ab,ti OR "intra-bony":ab,ti OR infrabony:ab,ti OR "infra-bony":ab,ti OR alveolar: ab,ti) AND [english]/lim.

CINAHL. (MH statins OR TI statin* OR AB statin* OR TI "reductase inhibitor*" OR AB "reductase inhibitor*" OR TI atorvastatin OR AB atorvastatin OR TI fluvastatin OR AB fluvastatin OR TI rosuvastatin OR AB rosuvastatin) AND ( $M H$ "periodontal diseases" OR MH "bone regeneration" OR TI periodont* OR AB periodont* OR TI "peri odontogy" OR AB "peri odontogy" OR TI mandible OR AB mandible OR TI "tooth extraction*" OR AB "tooth extraction*" OR TI furcation* OR AB furcation* OR TI intrabony complete outcome data; and 5) free of selective outcome reporting. Degree of bias was categorized as: 1 ) low risk if all criteria were met; 2 ) moderate risk when only one criterion was missing; and 3) high risk if two or more criteria were missing. Two reviewers (MPC and LS) assessed all included articles independently.

\section{Statistical Analyses}

Primary outcome was defect fill and secondary outcomes were PD reduction and CAL gain. Pooled weighted mean difference (WMD) of defect fill, PD reduction, and CAL gain between statin-treated group and control group were estimated using a computer program. ${ }^{\ddagger}$ To avoid potential bias of combining treatment outcomes of different types of statins, other statin-treated groups, such as simvastatin (SMV), rosuvastatin (RSV), or atorvastatin (ATV), were pooled as subgroup analyses. Contribution of each article was weighed. Inverse variance weighted (IV) effects meta-analyses of the selected studies were applied to avoid any bias caused by methodologic differences

\footnotetext{
‡ Review Manager (RevMan), Version 5.0, The Nordic Cochrane Center, The Cochrane Collaboration, Copenhagen, Denmark.
} 


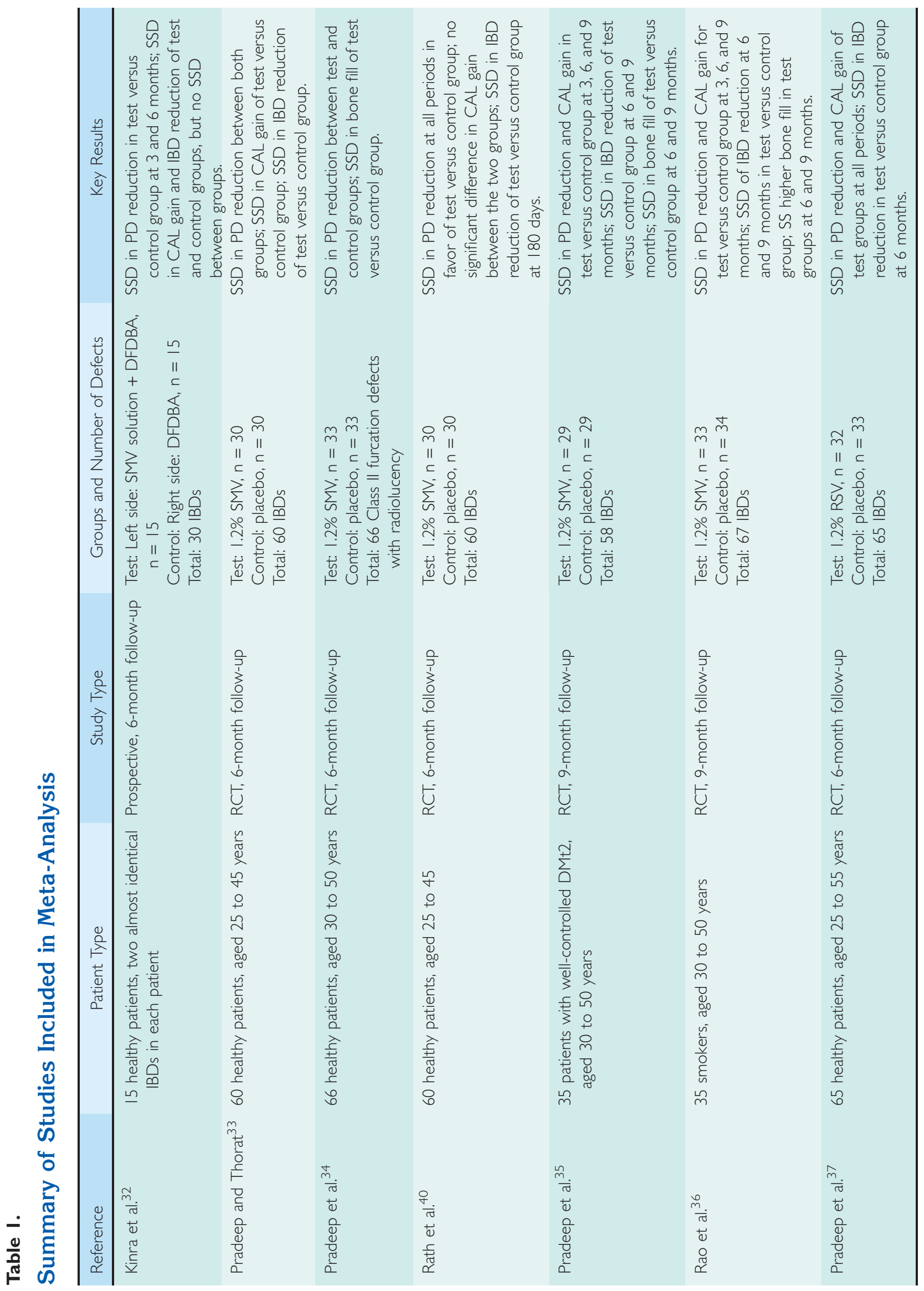




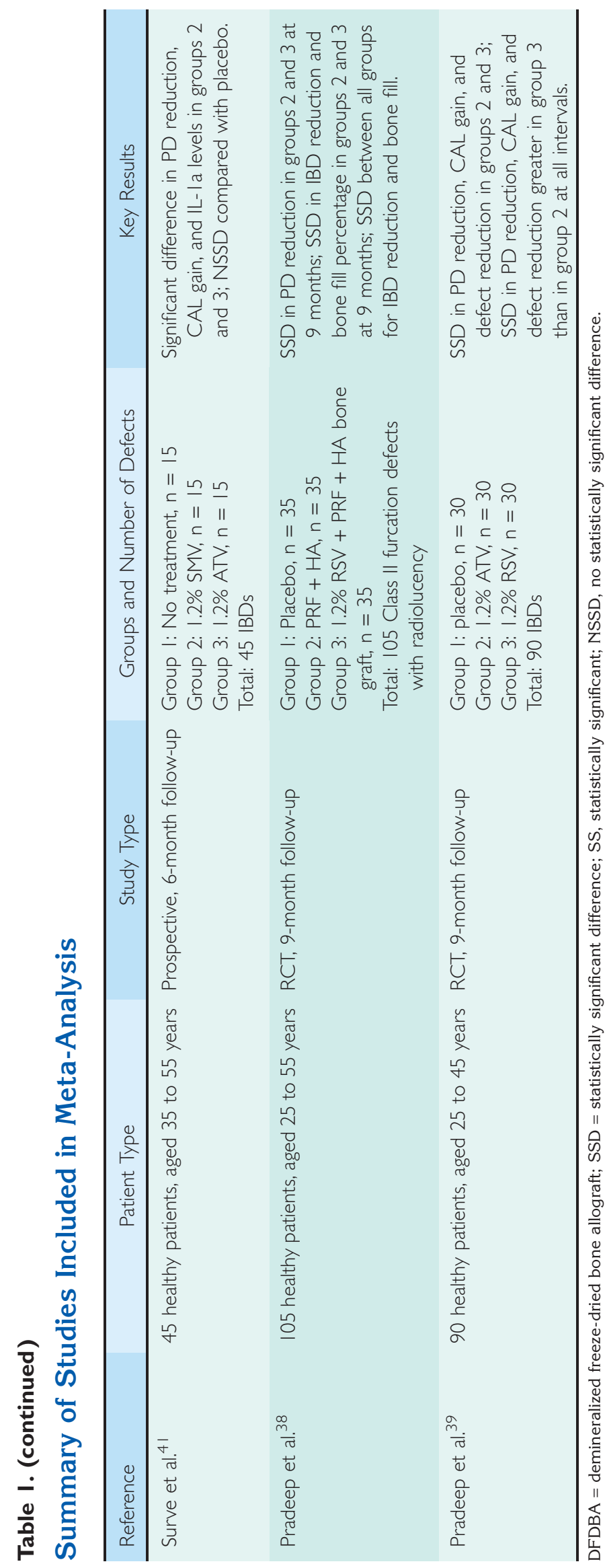

between studies. Forest plots were produced to graphically represent difference in outcomes of SMVtreated and placebo groups for all included studies using defect site as the analysis unit. Level of significance was set at $P \leq 0.05$. Heterogeneity was assessed with $\chi^{2}$ test and $\mathrm{I}^{2}$ test, which ranges between $0 \%$ and $100 \%$ with lower values representing less heterogeneity. In addition, funnel plot was used to assess presence of publication bias. Reporting of these meta-analyses adhered to the Preferred Reporting Items for Systematic Review and MetaAnalyses statement. ${ }^{31}$

\section{RESULTS}

\section{Results of Article Selection}

The search process is seen in Figure 1. The search yielded 204 articles through PubMed, 689 articles through Embase, and 54 articles through CINAHL (i.e., total 947 articles). An additional article was found manually because it was published after the search was completed, taking the total number of articles found to 948. Both examiners scanned all titles and abstracts for inclusion and exclusion criteria and identified 10 studies that fit the profile. ${ }^{32-41}$ After screening, examiners agreed on including all 10 studies. ${ }^{32-41}$ The $\kappa$ value was 0.856 with $95 \%$ confidence interval $(\mathrm{CI})$ of 0.65 to 1.00 . The full-text review $\kappa$ value was 1.00 . Number of participants in each study ranged between 35 and 105, with each group in a particular study consisting of 15 to 35 individuals (20 to 60 males and 0 to 50 females; age range 25 to 55 years; mean age: 40 years). Six studies had follow-up to 6 months and four of them to 9 months.

\section{Characteristics of Included Studies}

Each study included was different than the others (Table 1), despite seven of 10 belonging to the same group. ${ }^{33-39}$ In 2010, Kinra et al. ${ }^{32}$ compared effects of SMV on IBD when combined with an allograft in a prospective study. Pradeep and Thorat ${ }^{33}$ measured effects of SMV in treating IBD. In 2012, a study group focused on SMV effects in treating furcation involvements. ${ }^{34}$ In 2013, focus shifted from healthy to patients with well-controlled type 2 diabetes mellitus $(\mathrm{DMt} 2)^{35}$ and smokers. ${ }^{36}$ Afterward, RSV was used instead of SMV to calculate effectiveness of synthetic statins. ${ }^{37}$ Results of RSV proved it to be better than SMV and so in 2016, the same group decided to compare RSV results with ATV in one study, ${ }^{38}$ and when adding it to platelet-rich fibrin (PRF) and porous hydroxyapatite (HA) bone graft in another. ${ }^{39}$ Rath et al. ${ }^{40}$ evaluated effects of SMV on IL-6 levels as well as treatment outcomes. Surve et al. ${ }^{41}$ compared outcomes of SMV with those of ATV in another prospective study included in this meta-analysis. All other studies $33-40$ were RCTs that compared outcomes with a placebo 


\begin{tabular}{|c|c|c|c|c|c|c|c|c|c|c|}
\hline \multirow[b]{2}{*}{ Study or Subgroup } & \multicolumn{3}{|c|}{ Statins } & \multicolumn{3}{|c|}{ Control } & \multicolumn{2}{|r|}{ Mean Difference } & \multirow{2}{*}{\multicolumn{2}{|c|}{$\begin{array}{c}\text { Mean Difference } \\
\text { IV, Random; } 95 \% \mathrm{Cl}\end{array}$}} \\
\hline & Mean & SD & Total & Mean & SD & Total & Weight & IV, Random; 95\% Cl Year & & \\
\hline \multicolumn{11}{|l|}{ Simvastatin } \\
\hline Pradeep and Thorat 2010 & )$^{33} 1.41$ & 0.74 & 30 & 0.09 & 90.58 & 30 & $9.9 \%$ & $1.32[0.98,1.66] 2010$ & & \\
\hline Rath et al. $2012^{40}$ & 0.57 & 1 & 30 & 0.08 & $8 \quad 0.1$ & 30 & $9.8 \%$ & $0.49[0.13,0.85] 2012$ & & - \\
\hline Pradeep et al. $2012^{34}$ & 1.15 & 0.61 & 33 & 0.06 & 0.25 & 33 & $10.3 \%$ & $1.09[0.87,1.31] 2012$ & & - \\
\hline Pradeep et al. $2013^{35}$ & 1.62 & 0.71 & 29 & 0.23 & 30.49 & 29 & $10.0 \%$ & $1.39[1.08,1.70] 2013$ & & \\
\hline Rao et al. $2013^{36}$ & 1.51 & 0.47 & 33 & 0.21 & 10.27 & 34 & $10.4 \%$ & $1.30[1.12,1.48] 2013$ & & $\rightarrow$ \\
\hline $\begin{array}{l}\text { Surve et al. } 2015^{41} \\
\text { Subtotal }(95 \% \mathrm{Cl})\end{array}$ & 0.69 & 0.63 & $\begin{array}{r}15 \\
170\end{array}$ & 0.04 & 40.64 & $\begin{array}{r}15 \\
171\end{array}$ & $\begin{array}{r}9.4 \% \\
59.9 \%\end{array}$ & $\begin{array}{l}0.65[0.20,1.10] 2015 \\
1.07[0.81,1.33]\end{array}$ & & \\
\hline \multicolumn{11}{|c|}{$\begin{array}{l}\text { Heterogeneity: } \mathrm{Tau}^{2}=0.08 ; \mathrm{Chi}^{2}=23.63, \mathrm{df}=5(P=0.0003) ; I^{2}=79 \% \\
\text { Test for overall effect: } Z=8.12(P<0.00001)\end{array}$} \\
\hline \multicolumn{11}{|l|}{ Atorvastatin } \\
\hline Pradeep et al. $2016^{38}$ & 2.29 & 1.06 & 30 & 0.07 & 0.26 & 30 & $9.7 \%$ & $2.22[1.83,2.61] 2016$ & & \\
\hline $\begin{array}{l}\text { Surve et al. } 2015^{41} \\
\text { Subtotal }(95 \% \mathrm{Cl})\end{array}$ & 0.59 & 0.57 & $\begin{array}{l}15 \\
45\end{array}$ & 0.04 & 0.64 & $\begin{array}{l}15 \\
45\end{array}$ & $\begin{array}{r}9.5 \% \\
19.2 \%\end{array}$ & $\begin{array}{l}0.55[0.12,0.98] 2015 \\
1.39[-0.25,3.02]\end{array}$ & & \\
\hline \multicolumn{11}{|c|}{$\begin{array}{l}\text { Heterogeneity: } \mathrm{Tau}^{2}=1.35 ; \mathrm{Chi}^{2}=31.45, \mathrm{df}=1(P<0.00001) ;\left.\right|^{2}=97 \% \\
\text { Test for overall effect: } Z=1.66(P=0.10)\end{array}$} \\
\hline \multicolumn{11}{|l|}{ Rosuvastatin } \\
\hline Pradeep et al. $2016^{38}$ & 2.83 & 0.53 & 30 & 0.07 & 0.26 & 30 & $10.4 \%$ & $2.76[2.55,2.97] 2016$ & & \\
\hline $\begin{array}{l}\text { Pradeep et al. } 2015^{37} \\
\text { Subtotal }(95 \% \mathrm{Cl})\end{array}$ & 2.23 & 0.32 & $\begin{array}{l}32 \\
62\end{array}$ & 0.46 & 0.02 & $\begin{array}{l}33 \\
63\end{array}$ & $\begin{array}{l}10.6 \% \\
20.9 \%\end{array}$ & $\begin{array}{l}1.77[1.66,1.88] 2015 \\
2.26[1.29,3.23]\end{array}$ & & \\
\hline \multicolumn{11}{|c|}{$\begin{array}{l}\text { Heterogeneity: } \mathrm{Tau}^{2}=0.48 ; \mathrm{Chi}^{2}=66.09, \mathrm{df}=1(P<0.00001) ; I^{2}=98 \% \\
\text { Test for overall effect: } Z=4.57(P<0.00001)\end{array}$} \\
\hline \multirow{2}{*}{\multicolumn{8}{|c|}{$\begin{array}{l}\text { Total }(95 \% \mathrm{Cl}) \\
\text { Heterogeneity: } \text { Tau }^{2}=0.40 ; \mathrm{Chi}^{2}=244.67, \mathrm{df}= \\
\text { Test for overall effect: } \mathrm{Z}=6.59(P<0.00001)\end{array}$}} & $1.37[0.96,1.77]$ & & \\
\hline & & & & & & & & & $\begin{array}{ccc}1 & 1 & 1 \\
-2 & -1 & 0 \\
\text { Favors control }\end{array}$ & $\begin{array}{c}1 \\
\text { Favors sta }\end{array}$ \\
\hline
\end{tabular}

\section{Figure 2.}

Meta-analysis for comparison of defect fill among selected studies. WMD was 1.07, 1.39, and $2.26 \mathrm{~mm}$ for SMV, ATV, and RSV subgroups, respectively. Overall analysis presented WMD of $1.37 \mathrm{~mm}$, favoring statin-treated groups.

group. They used custom bite blocks for reproducibility and a computer-aided software program after scanning films to aid with measurements of defect bone fill. Surve et al. ${ }^{41}$ did not mention using custom bite blocks, but they did use conventional radiographs like all other studies.

\section{Results of Meta-Analyses for Defect Fill}

Seven studies ${ }^{32-36,40,41}$ reported data on defect fill of sites treated with SMV and placebo/no treatment. Two studies ${ }^{38,41}$ reported data on defect fill of sites treated with ATV and placebo/no treatment. Three studies ${ }^{37-39}$ reported data on defect fill of sites treated with RSV and placebo/no treatment. Statistical results from eight of the selected studies $33-38,40,41$ were converted into effect sizes and combined in the meta-analysis. Two studies ${ }^{32,} 39$ used a combination of bone graft with statins in the test group; therefore, these two studies were excluded from the meta-analyses due to incompatible data. WMD of defect fill was $1.07 \mathrm{~mm}(95 \% \mathrm{CI}=0.81$ to $1.33 ; P<0.0001)$, $1.39 \mathrm{~mm}(95 \% \mathrm{CI}=-0.25$ to $3.02 ; P=0.10)$, and $2.26 \mathrm{~mm}(95 \% \mathrm{CI}=1.29$ to $3.23 ; P<0.0001)$ for $\mathrm{SMV}$, ATV, and RSV subgroups, respectively (Fig. 2). Overall analysis presented WMD of $1.37 \mathrm{~mm}(95 \%$
$\mathrm{CI}=0.96$ to $1.77 ; P<0.0001)$, favoring statin-treated groups. However, comparison presented considerable heterogeneity among studies; $P$ values for $\chi^{2}$ test presented as $0.0003,<0.00001,<0.00001$, and $<0.00001$ for SMV, ATV, RSV, and overall subgroups, respectively.

\section{Results of Meta-Analyses for PD Reduction}

Seven studies $32-36,40,41$ reported data on PD reduction of sites treated with SMV and placebo/no treatment. Two studies 38,41 reported data on PD reduction of sites treated with ATV and placebo/no treatment. Three studies ${ }^{37-39}$ reported data on PD reduction of sites treated with RSV and placebo/no treatment. WMD of the eight studies included $33-38,40,41$ was $2.09 \mathrm{~mm}(95 \% \mathrm{CI}=1.05$ to $3.13 ; P<0.0001)$, $0.44 \mathrm{~mm}(95 \% \mathrm{CI}=-0.40$ to $1.28 ; P=0.30)$, and $2.15 \mathrm{~mm}(95 \% \mathrm{CI}=1.00$ to $3.30 ; P<0.0001)$ for $\mathrm{SMV}$, ATV, and RSV subgroups, respectively (Fig. 3). Overall analysis presented WMD of $1.76 \mathrm{~mm}(95 \%$ $\mathrm{CI}=1.04$ to $2.47 ; P<0.0001$ ), favoring statin-treated group. However, comparison presented considerable heterogeneity among studies; $P$ values for $\chi^{2}$ test presented as $<0.00001,<0.0002,<0.00001$, and $<0.00001$ for SMV, ATV, RSV, and overall subgroups, respectively. 


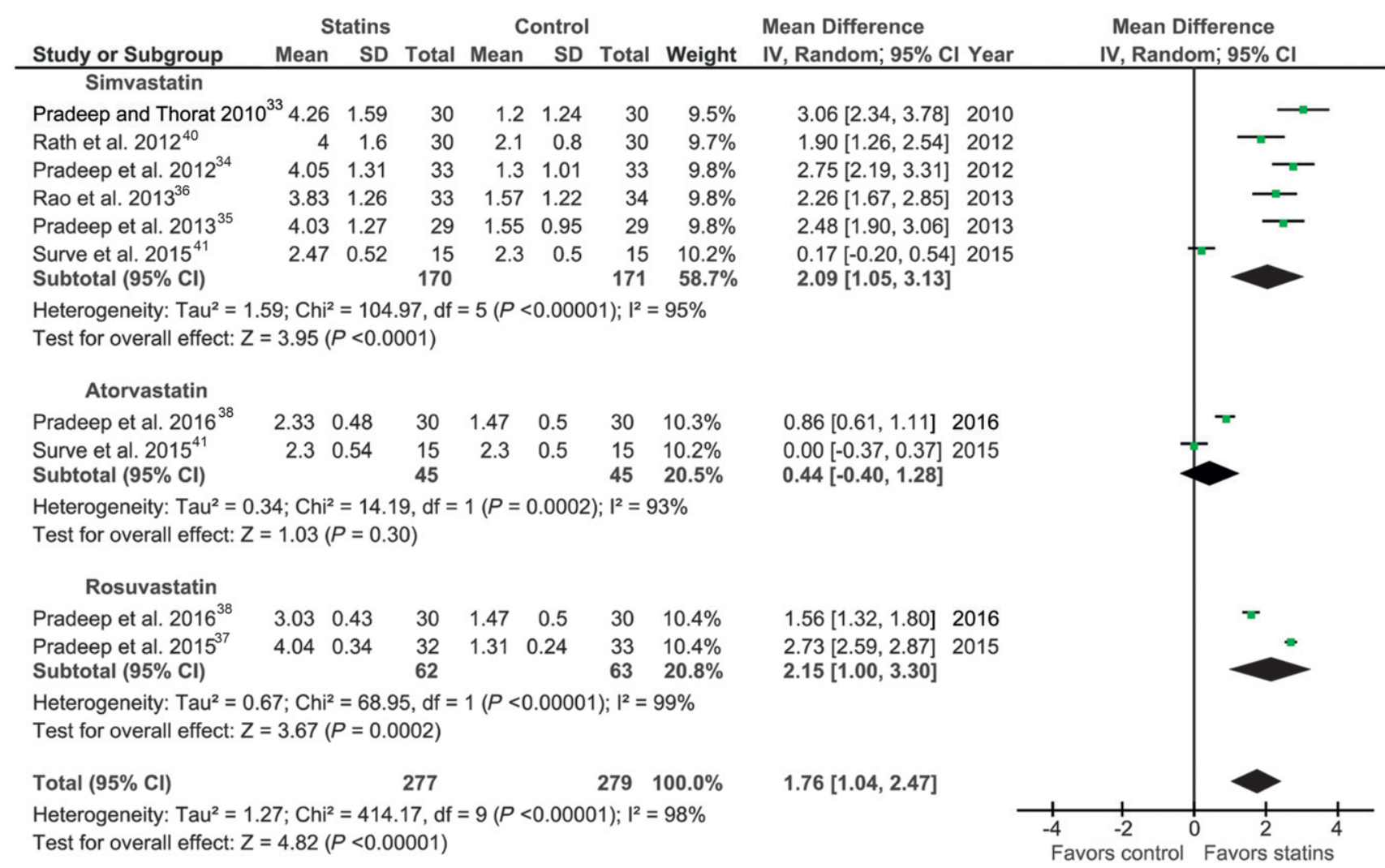

Figure 3.

Meta-analysis for comparison of PD reduction among selected studies. WMD was 2.09, 0.44, and $2.15 \mathrm{~mm}$ for SMV, ATV, and RSV subgroups, respectively. Overall analysis presented WMD of $1.76 \mathrm{~mm}$, favoring statin-treated groups.

\section{Results of Meta-Analyses for CAL Gain}

Seven studies ${ }^{32-36,40,41}$ reported data on CAL gain of sites treated with SMV and placebo/no treatment. Two studies ${ }^{38,41}$ reported data on CAL gain of sites treated with ATV and placebo/no treatment. Three studies $^{37-39}$ reported data on CAL gain of sites treated with RSV and placebo/no treatment. WMD of the eight studies included $33-38,40,41$ was $1.71 \mathrm{~mm}$ (95\% $\mathrm{Cl}=0.78$ to $2.64 ; P=0.0003), 0.67 \mathrm{~mm}(95 \% \mathrm{Cl}=$ -0.04 to $1.38 ; P=0.06)$, and $2.16 \mathrm{~mm}(95 \% \mathrm{CI}=$ 0.90 to $3.42 ; P=0.0008$ ) for SMV, ATV, and RSV subgroups, respectively (Fig. 4). Overall analysis presented WMD of $1.58 \mathrm{~mm}$ (95\% CI $=0.89$ to 2.28; $P<0.0001)$, favoring statin-treated group. However, comparison presented considerable heterogeneity among studies; $P$ values for $\chi^{2}$ test presented as $<0.00001,0.05,<0.00001$, and $<0.00001$ for SMV, ATV, RSV, and overall subgroups, respectively.

To investigate potential publication bias, funnel plots of meta-analyses for comparisons of defect fill, PD reduction, and CAL gain were demonstrated in supplementary Figures 1 (defect fill), 2 (PD reduction), and 3 (CAL gain) in online Journal of Periodontology. Due to the limited number of studies included, funnel plot could not be directly interpreted.
However, an asymmetric plot was noted and potential publication bias might exist.

\section{Results of Risk of Bias Assessment}

Results of risk of bias assessment for the included RCTs are summarized in Table 2. Eight studies $33-40$ were considered to have moderate risk of bias.

\section{DISCUSSION}

In the current review, three main statins identified among the studies included were SMV, ATV, and RSV. SMV is a natural, mevalonic acid-derived statin, and ATV is a synthetic, heptenoic acid-derived statin. SMV and ATV are both lipophilic, which is why they passively diffuse through the cell membrane and are transported by specific carriers. ${ }^{42}$ RSV is a hydrophilic statin that has prolonged effects compared with other statins. It works more selectively for hepatic cells than lipophilic statins, ${ }^{43}$ and is eight times more potent than pravastatin, another hydrophilic statin, and seven times more potent than ATV. ${ }^{44}$ RSV is also found to have greater anti-inflammatory action ${ }^{45}$ and is not significantly metabolized by cytochrome P450 enzymes like its lipophilic counterparts. ${ }^{46}$ Compared with lipophilic statins, RSV has been found to be 


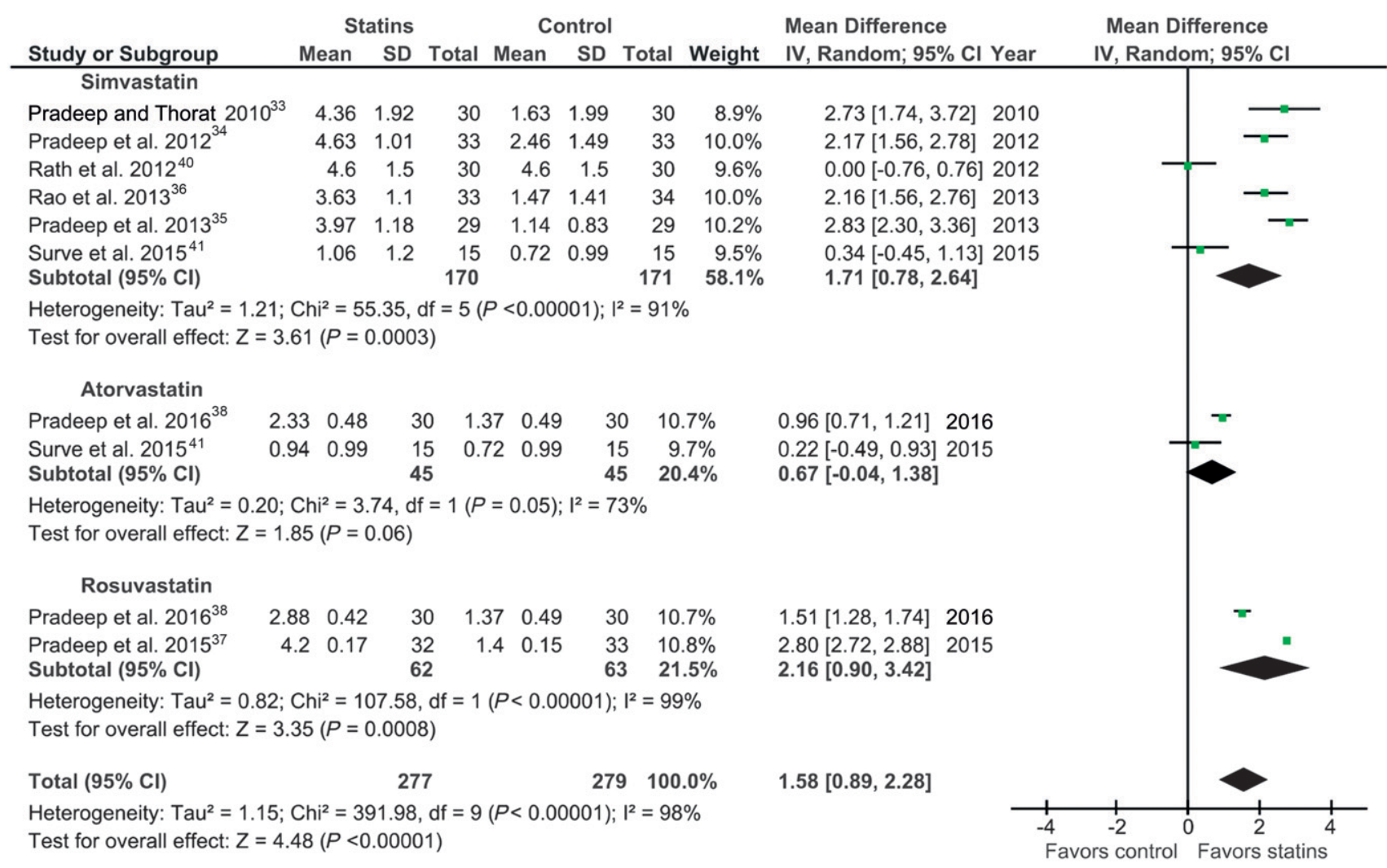

Figure 4.

Meta-analysis for comparison of CAL gain among selected studies. WMD was 1.71, 0.67, and 2.16 mm for SMV, ATV, and RSV subgroups, respectively. Overall analysis presented WMD of $1.58 \mathrm{~mm}$, favoring statin-treated groups.

superior in the reversion of coronary atherosclerotic plaques, ${ }^{47}$ in lowering low-density lipoprotein cholesterol, ${ }^{48,} 49$ and in reduction of small, dense lowdensity lipoproteins. ${ }^{50}$ It has also been proved that hydrophilic statins in general are less deadly than lipophilic statins. ${ }^{51}$

Results obtained from this study showed statintreated groups having significant defect fill (WMD of $1.37 \mathrm{~mm}$ ), PD reduction (WMD of $1.76 \mathrm{~mm}$ ), and CAL gain (WMD of $1.58 \mathrm{~mm}$ ) compared with placebo/no treatment control groups. This can be explained by the anti-inflammatory property that statins possess. For example, SMV has been found to decrease production of IL- 6 and IL-8, proving antiinflammatory properties of statins. ${ }^{26} \mathrm{~A}$ similar finding was also reported by Rath et al. ${ }^{40}$, where the SMVtreated group showed mean decrease in IL- 6 level from day 0 to day 90 , but not in the placebo group. Lindy et al. ${ }^{13}$ compared treatment outcome of patients taking statins with those who did not take statins and demonstrated that patients with periodontitis who also took statin drugs had 37\% fewer pathologic periodontal pockets than those not taking statin medication. The authors elaborated beneficial effects of statins to be mediated by their pleiotropic anti-inflammatory effect on periodontal tissue. Similarly, in multiple studies by Pradeep et al., ${ }^{33-35}$ reduction in bleeding index from baseline to 6 months was obvious when using SMV as a result of its antiinflammatory properties.

Clinical benefits of using locally delivered statins were compared with those of using locally delivered antibiotics. According to the systematic review on local anti-infective therapy by Hanes and Purvis, ${ }^{6}$ WMD for PD reduction was $0.35,0.51,0.06,0.36$, 0.26 , and $0.21 \mathrm{~mm}$ for CHX, DOX gels, MET, MIN, MIN microspheres, and TCN fibers, respectively. PD reduction WMD for statins was 2.09, 0.44, and $2.15 \mathrm{~mm}$ for SMV, ATV, and RSV, respectively. For CAL gain, WMD was $0.16,0.34,0.07,0.39,-0.40$, and $-0.17 \mathrm{~mm}$ for CHX, DOX gels, MET, MIN, MIN microspheres, and TCN fibers, respectively. CAL gain WMD for statins was $1.71,0.67$, and $2.16 \mathrm{~mm}$ for SMV, ATV, and RSV, respectively. Range of PD reduction and CAL gain in statins is much greater than locally delivered antimicrobials. However, further RCTs on statins are needed for a more definitive comparison.

All the selected studies used locally delivered statins instead of systemically delivered ones. This 


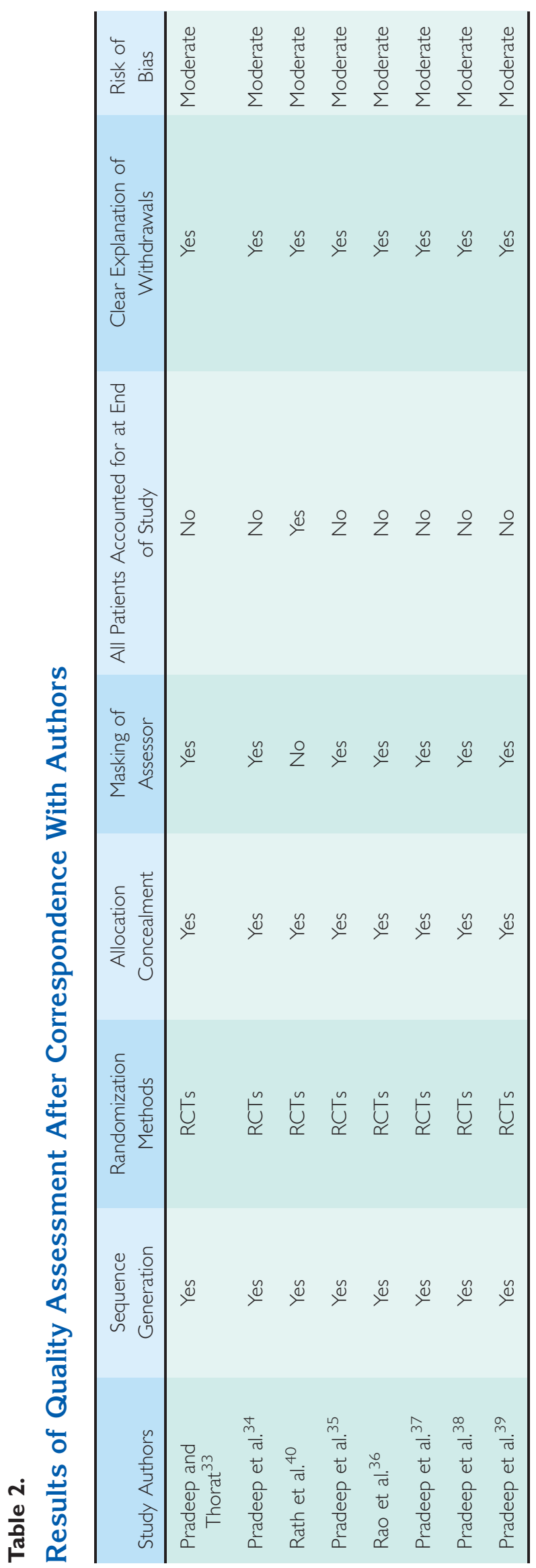

preference was made because of their rapidly absorbed characteristic after oral administration and their low bioavailability due to their high liver specificity. ${ }^{46}$ They were also found to be less effective when given systemically due to their higher liver clearance. $^{52}$ The subgingival drug-delivery system also achieves higher intrasulcular drug concentrations, avoids systemic adverse effects, and attains better patient compliance. ${ }^{53}$ Side effects from taking statins include myopathy, myalgia, rhabdomyolysis, and elevated liver function tests that could possibly lead to liver damage. ${ }^{54}$ However, these adverse events are rare when given at standard doses ${ }^{55}$ and, to the best of the authors' knowledge, none of the studies using gel-form statins have yet reported complications or adverse reactions. As for locally delivered antibiotics, a systematic review found mild side effects to be reported in different studies. Some of these side effects include pain during agent placement, site-specific gingival redness, gingival abscesses, root sensitivity, oral candidiasis with some TCN fiber groups, and toothache and headache with some MIN and CHX groups. ${ }^{6}$

Other modifying factors in treating IBDs, such as DM and smoking, were taken into consideration in some studies. Only one selected study ${ }^{35}$ in the current meta-analyses included patients with wellcontrolled DMt2 in the RCT. However, results after treatment showed PD reduction and bone gain similar to patients in all other studies not including healthy patients. This outcome was consistent with previous findings as DM was well controlled so that host defense cells were functioning similar to those of healthy patients. ${ }^{56}$ Regarding smoking, Rao et al. ${ }^{36}$ included smokers in their RCT. All participants smoked $>10$ cigarettes/day for more than 5 years. Interestingly, results after treatment presented PD reduction and bone gain similar to patients who were not smokers. This outcome might result from the different amounts of nicotine taken and inclusion criteria of the study design. ${ }^{36}$

Limitations noted in the current study were: 1) possibility of selection bias as most of the included studies (seven of 10) were from the same study groups; ${ }^{33-39} 2$ ) relatively small sample sizes of the included studies, ranging from 15 to 35 participants per test/control group; 3) accuracy of measurements was not calibrated in most of the studies, which might contribute to geometric errors in IBD fill on conventional radiographs; 4) various study designs and inclusion/exclusion criteria among the selected studies; and 5) search criteria involved only English-language articles. Therefore, results of the current review should be interpreted cautiously. 


\section{CONCLUSIONS}

Adjunctive use of locally delivered statins with nonsurgical periodontal treatment in IBDs shows promising results in bone fill, reduction of inflammation and bleeding, PD reduction, and CAL gain. These results, compared with adjunctive use of locally delivered antimicrobials, were superior in PD reduction and CAL gain. There is need for future multicenter RCTs to warrant additional benefits of using statins in combination with non-surgical periodontal treatment for patients with periodontitis.

\section{ACKNOWLEDGMENTS}

The authors do not have any financial interests, either directly or indirectly, in the products or information listed in the paper. KS was responsible for manuscript preparation and data interpretation; NZ for article search and selection; G-HL for statistics and manuscript preparation; M-PC for article selection and risk bias assessment; and LS for risk bias assessment and analysis of article references. H-LW was study supervisor and advisor. This paper was partially supported by the University of Michigan Periodontal Graduate Student Research Fund. The authors report no conflicts of interest related to this study.

\section{REFERENCES}

1. Slots J. Periodontology: Past, present, perspectives. Periodontol 2000 2013;62:7-19.

2. Shimizu H, Nakagami H, Morita S, et al. New treatment of periodontal diseases by using NF-kappaB decoy oligodeoxynucleotides via prevention of bone resorption and promotion of wound healing. Antioxid Redox Signal 2009;11:2065-2075.

3. Seymour GJ, Gemmell E, Reinhardt RA, Eastcott J, Taubman MA. Immunopathogenesis of chronic inflammatory periodontal disease: Cellular and molecular mechanisms. J Periodontal Res 1993;28:478-486.

4. Addy M, Renton-Harper P. Local and systemic chemotherapy in the management of periodontal disease: An opinion and review of the concept. JOral Rehabil 1996; 23:219-231.

5. American Academy of Periodontology. American Academy of Periodontology statement on local delivery of sustained or controlled release antimicrobials as adjunctive therapy in the treatment of periodontitis. $J$ Periodontol 2006;77:1458.

6. Hanes PJ, Purvis JP. Local anti-infective therapy: Pharmacological agents. A systematic review. Ann Periodontol 2003;8:79-98.

7. Goldstein JL, Brown MS. Regulation of the mevalonate pathway. Nature 1990;343:425-430.

8. Medscape. 100 Best-selling, most prescribed branded drugs through March. May 2015. Available at: http:// www.medscape.com/viewarticle/844317\#vp_1. Accessed April 15, 2016.

9. Endres M. Statins: Potential new indications in inflammatory conditions. Atheroscler Suppl 2006;7:31-35.

10. Adam O, Laufs U. Antioxidative effects of statins. Arch Toxicol 2008;82:885-892.

11. Jerwood S, Cohen J. Unexpected antimicrobial effect of statins. J Antimicrob Chemother 2008;61:362-364.
12. Forrester JS, Libby P. The inflammation hypothesis and its potential relevance to statin therapy. Am J Cardiol 2007;99:732-738.

13. Lindy O, Suomalainen K, Mäkelä M, Lindy S. Statin use is associated with fewer periodontal lesions: A retrospective study. BMC Oral Health 2008;8:16.

14. Sundararaj KP, Samuvel DJ, Li Y, et al. Simvastatin suppresses LPS-induced MMP-1 expression in U937 mononuclear cells by inhibiting protein isoprenylationmediated ERK activation. J Leukoc Biol 2008;84: 1120-1129.

15. Thunyakitpisal PD, Chaisuparat R. Simvastatin, an HMG-CoA reductase inhibitor, reduced the expression of matrix metalloproteinase-9 (gelatinase B) in osteoblastic cells and HT1080 fibrosarcoma cells. JPharmacol Sci 2004;94:403-409.

16. Li JJ, Chen XJ. Simvastatin inhibits interleukin-6 release in human monocytes stimulated by $\mathrm{C}$-reactive protein and lipopolysaccharide. Coron Artery Dis 2003; 14:329-334.

17. Rosenson RS, Tangney CC, Levine DM, Parker TS, Gordon BR. Association between reduced low density lipoprotein oxidation and inhibition of monocyte chemoattractant protein-1 production in statin-treated subjects. J Lab Clin Med 2005;145:83-87.

18. Takeuchi S, Kawashima S, Rikitake Y, et al. Cerivastatin suppresses lipopolysaccharide-induced ICAM-1 expression through inhibition of Rho GTPase in BAEC. Biochem Biophys Res Commun 2000;269:97-102.

19. Huang KC, Chen CW, Chen JC, Lin WW. Statins induce suppressor of cytokine signaling-3 in macrophages. FEBS Lett 2003;555:385-389.

20. Grasser WA, Baumann AP, Petras SF, et al. Regulation of osteoclast differentiation by statins. JMusculoskelet Neuronal Interact 2003;3:53-62.

21. Yazawa H, Zimmermann B, Asami Y, Bernimoulin JP. Simvastatin promotes cell metabolism, proliferation, and osteoblastic differentiation in human periodontal ligament cells. J Periodontol 2005;76:295-302.

22. Maeda T, Matsunuma A, Kurahashi I, Yanagawa T, Yoshida $\mathrm{H}$, Horiuchi N. Induction of osteoblast differentiation indices by statins in MC3T3-E1 cells. $J$ Cell Biochem 2004;92:458-471.

23. Stein D, Lee Y, Schmid MJ, et al. Local simvastatin effects on mandibular bone growth and inflammation. $J$ Periodontol 2005;76:1861-1870.

24. Nyan M, Sato D, Oda $M$, et al. Bone formation with the combination of simvastatin and calcium sulfate in critical-sized rat calvarial defect. $J$ Pharmacol Sci 2007; 104:384-386.

25. Goes P, Lima AP, Melo IM, Rêgo RO, Lima V. Effect of atorvastatin in radiographic density on alveolar bone loss in wistar rats. Braz Dent J 2010;21:193-198.

26. Sakoda K, Yamamoto M, Negishi Y, Liao JK, Node K, Izumi Y. Simvastatin decreases IL- 6 and IL- 8 production in epithelial cells. J Dent Res 2006;85:520-523.

27. Jordan E, Hsu YT, Bashutski J. Do statin medications improve periodontal health and/or outcomes? A systematic review. Clinic Adv Periodontics 2014;4:194202.

28. Estanislau IM, Terceiro IR, Lisboa MR, et al. Pleiotropic effects of statins on the treatment of chronic periodontitis - A systematic review. Br J Clin Pharmacol 2015; 79:877-885.

29. Higgins JPT, Green S. Handbook for systematic reviews of interventions version 5.1. 0 [updated March 2011]. The Cochrane Collaboration 2011. Available 
at: http://handbook.cochrane.org/. Accessed April 1, 2016.

30. Schulz KF, Altman DG, Moher D. CONSORT 2010 statement: Updated guidelines for reporting parallel group randomised trials. J Pharmacol Pharmacother 2010;1:100-107.

31. Liberati A, Altman DG, Tetzlaff J, et al. The PRISMA statement for reporting systematic reviews and metaanalyses of studies that evaluate health care interventions: Explanation and elaboration. Ann Intern Med 2009;151:W65-W94.

32. Kinra P, Gupta H, Khan S, Ahmad MS. Evaluation of the relative efficacy of an allograft used alone and that in combination with simvastatin in the treatment of human periodontal infrabony defects: A clinical and radiological study. J T U Med Sc 2010;5:75-88.

33. Pradeep AR, Thorat MS. Clinical effect of subgingivally delivered simvastatin in the treatment of patients with chronic periodontitis: A randomized clinical trial. $J$ Periodontol 2010;81:214-222.

34. Pradeep AR, Priyanka N, Kalra N, Naik SB, Singh SP, Martande S. Clinical efficacy of subgingivally delivered 1.2 -mg simvastatin in the treatment of individuals with class II furcation defects: A randomized controlled clinical trial. J Periodontol 2012;83:1472-1479.

35. Pradeep AR, Rao NS, Bajaj P, Kumari M. Efficacy of subgingivally delivered simvastatin in the treatment of patients with type 2 diabetes and chronic periodontitis: A randomized double-masked controlled clinical trial. $J$ Periodontol 2013;84:24-31.

36. Rao NS, Pradeep AR, Bajaj P, Kumari M, Naik SB. Simvastatin local drug delivery in smokers with chronic periodontitis: A randomized controlled clinical trial. Aust Dent J 2013;58:156-162.

37. Pradeep AR, Karvekar S, Nagpal K, Patnaik K, Guruprasad CN, Kumaraswamy KM. Efficacy of locally delivered $1.2 \%$ rosuvastatin gel to non-surgical treatment of patients with chronic periodontitis: A randomized, placebo-controlled clinical trial. J Periodontol 2015; 86:738-745.

38. Pradeep AR, Garg V, Kanoriya D, Singhal S. $1.2 \%$ rosuvastatin versus $1.2 \%$ atorvastatin gel local drug delivery and redelivery in treatment of intrabony defects in chronic periodontitis: A randomized placebo-controlled clinical trial. J Periodontol 2016;87: 756-762.

39. Pradeep AR, Karvekar S, Nagpal K, Patnaik K, Raju A, Singh P. Rosuvastatin $1.2 \mathrm{mg}$ in situ gel combined with $1: 1$ mixture of autologous platelet-rich fibrin and porous hydroxyapatite bone graft in surgical treatment of mandibular class II furcation defects: A randomized clinical control trial. J Periodontol 2016;87:5-13.

40. Rath A, Mahenra J, Thomas L, Sandhu M, Namasi A, Ramakrishna T. A clinical, radiological and IL- 6 evaluation of subgingivally delivered simvastatin in the treatment of chronic periodontitis. Int J Drug Deliv 2012;4:70.

41. Surve SM, Acharya AB, Thakur SL. Efficacy of subgingivally delivered atorvastatin and simvastatin as an adjunct to scaling and root planing. Drug Metab Pers Ther 2015;30:263-269.

42. Bonsu KO, Kadirvelu A, Reidpath DD. Lipophilic versus hydrophilic statin therapy for heart failure: A protocol for an adjusted indirect comparison meta-analysis. Syst Rev 2013;2:22.

43. Luvai A, Mbagaya W, Hall AS, Barth JH. Rosuvastatin: A review of the pharmacology and clinical effectiveness in cardiovascular disease. Clin Med Insights Cardiol 2012;6:17-33.

44. McTaggart F, Buckett L, Davidson R, et al. Preclinical and clinical pharmacology of rosuvastatin, a new 3hydroxy-3-methylglutaryl coenzyme $A$ reductase inhibitor. Am J Cardiol 2001;87:28B-32B.

45. Ma L, Wang Y, Du J, Wang M, Zhang R, Fu Y. The association between statin use and risk of age-related macular degeneration. Sci Rep 2015;5:18280.

46. Schachter M. Chemical, pharmacokinetic and pharmacodynamic properties of statins: An update. Fundam Clin Pharmacol 2005;19:117-125.

47. Qian C, Wei B, Ding J, et al. Meta-analysis comparing the effects of rosuvastatin versus atorvastatin on regression of coronary atherosclerotic plaques. Am J Cardiol 2015;116:1521-1526.

48. McKenney JM, Jones PH, Adamczyk MA, Cain VA, Bryzinski BS, Blasetto JW; STELLAR Study Group. Comparison of the efficacy of rosuvastatin versus atorvastatin, simvastatin, and pravastatin in achieving lipid goals: Results from the STELLAR trial. Curr Med Res Opin 2003;19:689-698.

49. Shioji K, Izuhara M, Mitsuoka H, Uegaito T, Matsuda M; Kishiwada Atherosclerosis Prevention Study (KAPS) Group. Achievement rates of Japan Atherosclerosis Society Guidelines 2007 LDL-cholesterol goals with rosuvastatin or atorvastatin in patients who had not achieved their goal with atorvastatin. Cardiovasc Ther 2014;32:97-104.

50. Takagi H, Niwa M, Mizuno Y, Yamamoto H, Goto SN, Umemoto T. Effects of rosuvastatin versus atorvastatin on small dense low-density lipoprotein: A meta-analysis of randomized trials. Heart Vessels 2014;29:287-299.

51. Sakamoto K, Mikami H, Kimura J. Involvement of organic anion transporting polypeptides in the toxicity of hydrophilic pravastatin and lipophilic fluvastatin in rat skeletal myofibres. Br J Pharmacol 2008;154:1482-1490.

52. Todd PA, Goa KL. Simvastatin. A review of its pharmacological properties and therapeutic potential in hypercholesterolaemia. Drugs 1990;40:583-607.

53. Goodson JM, Offenbacher S, Farr DH, Hogan PE. Periodontal disease treatment by local drug delivery. $J$ Periodontol 1985;56:265-272.

54. Silva MA, Swanson AC, Gandhi PJ, Tataronis GR. Statin-related adverse events: A meta-analysis. Clin Ther 2006;28:26-35.

55. Armitage J. The safety of statins in clinical practice. Lancet 2007;370:1781-1790.

56. Tervonen $\mathrm{T}$, Knuuttila $\mathrm{M}$, Pohjamo L, Nurkkala $\mathrm{H}$. Immediate response to nonsurgical periodontal treatment in subjects with diabetes mellitus. J Clin Periodontol 1991;18:65-68.

Correspondence: Prof. Hom-Lay Wang, 1011 N. University Ave., Ann Arbor, MI 48109-1078. Fax: (734)-936-0374; e-mail: homlay@umich.edu.

Submitted June 13, 2016; accepted for publication October 1, 2016. 